\title{
Prevalência dos casos de sífilis em gestantes no período de 2010 a 2019 em Sergipe
}

\author{
Prevalence of syphilis cases in pregnant women from 2010 to 2019 in Sergipe \\ Prevalencia de casos de sífilis en mujeres embarazadas de 2010 a 2019 en Sergipe
}

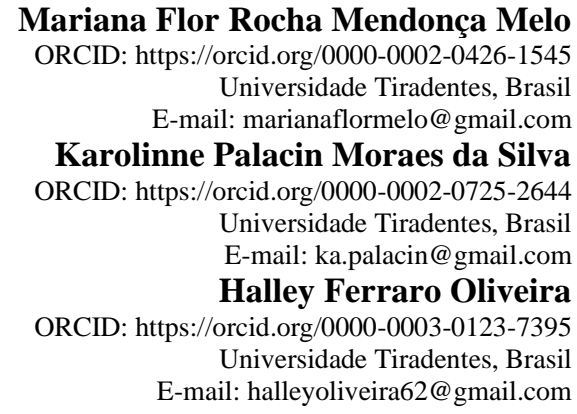

\begin{abstract}
Resumo
Objetivo: Delinear o perfil epidemiológico de mulheres positivas para a sífilis durante a gestação no estado de Sergipe no período de dez anos. Métodos: Estudo transversal retrospectivo realizado entre 2010 e 2019. Os dados foram obtidos no DATASUS. Todos os cálculos foram realizados com o software Excel do próprio banco de dados. As variáveis selecionadas incluídas foram: infecção de sífilis na gestação, escolaridade, etnia, faixa etária e idade gestacional. Resultados: Houve um aumento do quantitativo dos casos de sífilis em gestantes do período de 2010 a 2019, com aumento de, aproximadamente, $17,1 \%$ ao ano. Foi encontrado que $74,8 \%$ dos casos ocorreram em mulheres são pardas, 50,3\% em mulheres na faixa etária dos 20 a 29 anos, $27 \%$ em mulheres apresentam nível educacional da $5^{\mathrm{a}}$ a $8^{\mathrm{a}}$ série incompleta e $38 \%$ dos casos foi diagnosticado no terceiro trimestre de gestação. Conclusão: Este estudo apresentou o crescimento dos casos de sífilis em gestantes no estado de Sergipe na última década, sendo predominante em mulheres pardas, jovens e com baixa escolaridade.
\end{abstract}

Palavras-chave: Doença infectocontagiosa; IST; Sífilis; Gravidez de alto risco; Doença de notificação compulsória; Cuidado pré-natal.

\begin{abstract}
Objective: To delineate the epidemiological profile of women positive for syphilis during pregnancy in the state of Sergipe in ten years. Method: Retrospective cross-sectional study conducted between 2010 and 2019. The data were obtained from DATASUS. All calculations were performed with Excel software from the database itself. The selected variables included were: syphilis infection in pregnancy, education, ethnicity, age group, and gestational age. Results: There was an increase in the number of syphilis cases in pregnant women from 2010 to 2019, with an increase of approximately $17.1 \%$ per year. It was found that $74.8 \%$ of cases occurring in brown women, $50.3 \%$ in women in the age group of 20 to 29 years old, $27 \%$ in women with an educational level of 5th to 8th grade incomplete, and $38 \%$ of cases were diagnosed in the third trimester of pregnancy. Conclusion: This study presented the growth of syphilis cases in pregnant women in the state of Sergipe in the last decade, predominantly in brown, young, and poorly educated women.
\end{abstract}

Keywords: Infectious disease; STI; Syphilis; High-risk pregnancy; Disease notifications; Prenatal care.

\section{Resumen}

Delinear el perfil epidemiológico de las mujeres positivas a la sífilis durante el embarazo en el estado de Sergipe en un período de diez años. Método: Estudio transversal retrospectivo realizado entre 2010 y 2019. Los datos se obtuvieron de DATASUS. Todos los cálculos se realizaron con el software Excel de la propia base de datos. Las variables seleccionadas fueron: infección por sífilis en el embarazo, educación, etnia, grupo de edad y edad gestacional. Resultados: Hubo un aumento en los casos cuantitativos de sífilis en mujeres embarazadas desde 2010 hasta 2019 , con un aumento de aproximadamente el $17,1 \%$ por año. Se encontró que el $74,8 \%$ de los casos se produjo en las mujeres son de color marrón, el 50,3\% en las mujeres en el grupo de edad de 20 a 29 años, el $27 \%$ en las mujeres presentan nivel educativo de $5^{\circ}$ a $8^{\circ}$ grado incompleto y el $38 \%$ de los casos fue diagnosticado en el tercer trimestre del embarazo. Conclusión: Este estudio mostró el crecimiento de los casos de sífilis en mujeres embarazadas en el estado de Sergipe en la última década, predominantemente en mujeres morenas, jóvenes y con baja educación. 
Palabras clave: Enfermedad infecciosa; ITS; Sífilis; Embarazo de alto riesgo; Notificación de enfermedades; Atención prenatal.

\section{Introdução}

A sífilis é uma doença infectocontagiosa e sistêmica, a qual pode ser classificada como uma infecção sexualmente transmissível (IST). Ela é causada pelo Treponema pallidum, uma bactéria espiroqueta, um dos vários treponemas intimamente relacionados que causam doenças humanas. A transmissão geralmente ocorre através do contato direto com uma lesão infecciosa durante a relação sexual. A transmissão sanguínea pode ocorrer, contudo é mais rara, ademais o T. pallidum cruza prontamente a placenta, resultando assim em infecção fetal. (Musher, 1990)

Sua incidência aumentou significativamente no Brasil e no mundo nos últimos anos. Nos Estados Unidos, de 2000 a 2018, o aumento na taxa de casos relatados de sífilis primária e secundária foi principalmente atribuível ao aumento de casos em homens que fazem sexo com homens (HSH). Os HSH representavam aproximadamente 54\% de todos os casos de sífilis primária e secundária em 2018. (CDC, 2018)

Em se tratando da sífilis gestacional, ela constitui um problema de saúde pública no mundo. (Arnesen, 2015). Em torno de $90 \%$ dos casos ocorrem em países em desenvolvimento; entretanto, têm-se observado o reaparecimento em nações desenvolvidas. (Cameron, 2014). Em 2011, a Organização Mundial da Saúde (OMS) estimou que, anualmente, ocorram 2 milhões de casos de sífilis em gestantes sendo cerca de $25 \%$ dos casos não tratados, ou tratados inadequadamente, os quais resultariam em um aborto espontâneo ou natimortos.

A doença provoca múltiplos desfechos adversos na gravidez, sendo estimado um risco de 4,5 vezes maior, quando comparados às gestantes sem o diagnóstico (Gomez, 2013). Além disso, os recém-nascidos de mães com sífilis não tratada ou tratada inadequadamente podem apresentar quadro assintomático ao nascer. Isso, pode levar à ausência de diagnóstico e tratamento neonatal, gerando graves danos à sua saúde, com repercussões psicológicas e sociais. (Casal, 2011)

No Brasil, a vigilância dessa infecção é considerada compulsória desde 2005, mediante registro de notificação no Sistema de Informação de Agravos de Notificação (Sinan). Ademais, em 2011 foi instituída a Rede Cegonha, uma importante rede de ações e serviços com o objetivo de oferecer atenção humanizada à gravidez, parto e puerpério, garantir o direito ao nascimento seguro e ao crescimento e desenvolvimento saudáveis. A implantação da Rede Cegonha trouxe a expansão da triagem pré-natal da sífilis, por meio da descentralização dos testes rápidos para a Atenção Básica à Saúde, ampliando a capacidade de detecção.

Devido a relevância desse tema no impacto da saúde materno-fetal, em Sergipe, a Rede Cegonha foi implantada pela Portaria no 3.069 GAB/MS de 27 de dezembro de 2012. Com isso, de acordo com o Plano Estadual de Saúde, a sífilis em gestante nos últimos anos apresentou-se com elevação no número de notificações. Dessa forma, a implementação dessa política já se mostrou benéfica, uma vez que essa elevação já é um reflexo do fortalecimento das ações implementadas na atenção básica com a testagem das gestantes no momento da consulta pré-natal. (Ministério da Saúde, 2016).

O presente trabalho tem como objetivo delinear o perfil epidemiológico de mulheres positivas para a sífilis durante a gestação no estado de Sergipe, no período de dez anos e trazer propostas para diminuição destes casos.

\section{Metodologia}

Estudo transversal, retrospectivo e histórico realizado entre 2010 a 2019. Os dados epidemiológicos foram obtidos no banco de dados do Departamento de Informática do SUS (DATASUS), disponibilizadas pelo do Sistema Nacional de Agravos de Notificação (SINAN), oriundos do formulário "Doenças e Agravos de Notificação", documento este preenchido por qualquer profissional de saúde que suspeitou ou confirmou a sífilis em gestantes. 
A categorização das variáveis "infecção de sífilis na gestação", "escolaridade”, "etnia", "faixa etária" e "idade gestacional" foram realizadas pelo TABNET. A análise estatística, as operações aritméticas bem como a elaboração dos gráficos e tabelas foram executadas pelo TABWIN. Segue em fluxograma (Figura 1), o passo-a-passo da coleta de dados, no Departamento de informática do SUS, disponibilizadas no seguinte endereço eletrônico: Indicadores Sífilis - DCCI (aids.gov.br)<(acesso: fevereiro de 2021).

Figura 1. Fluxograma da coleta de dados em DATASUS.

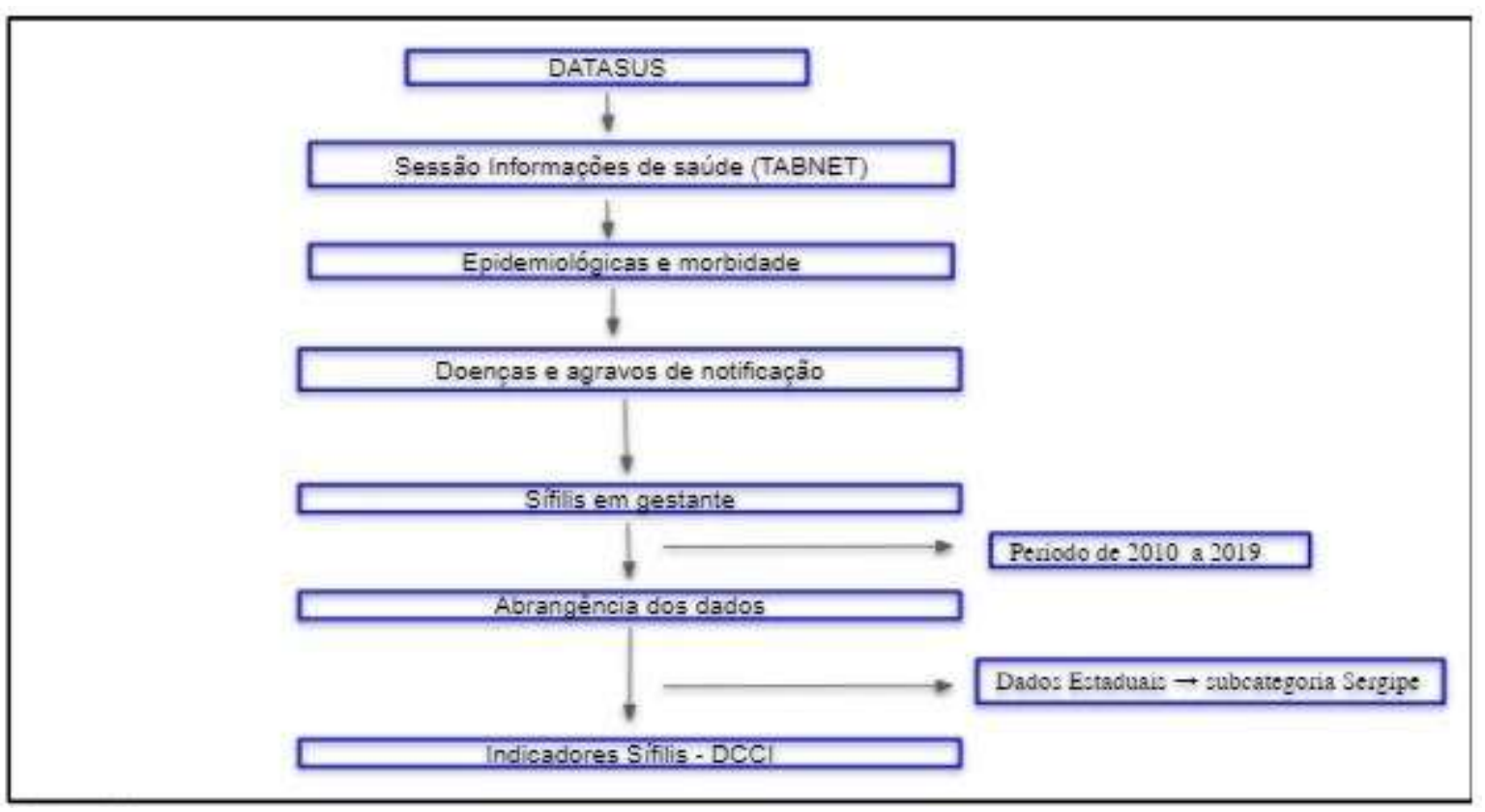

Fonte: Autores.

\section{Resultados}

No período de 2010 a 2019, foram notificados pelo SINAN de Sergipe, 3.865 casos de sífilis em gestantes. No ano de 2010, foram 178 novos casos com taxa de detecção de 5,2/1.000 nascidos vivos (NV). No ano de 2011, o número subiu para 312 casos, sendo a taxa de detecção de 8,9/ 1.000 NV. Em 2012, o número de casos foi parar 326 com taxa de detecção de 9,6/1.000 NV. Em 2013, ocorreu uma menor taxa de detecção: 7,6/1.000 NV e 259 casos de sífilis.

Em 2014, ocorreram 303 casos, com taxa de 8,8/ 1.000 NV. Em 2015, houve um aumento discreto, apresentando 341 casos com taxa de 9,8/1.000 NV. Em 2016, a taxa e o número de casos caíram levemente, com 305 casos com taxa de 9,5/1.000 NV. Em 2017, os números voltaram a progredir, com 447 casos com taxa de 13,2/1.000 NV. Em 2018 ocorreram 653 casos com taxa de 19,1/1.000 NV e, por fim, no ano de 2019, foram notificados 741 casos com taxa de 21,6/1.000 NV.

Com isso, o ano de 2019 apresentou-se com maior número de casos notificados de sífilis gestacional, evidenciando um aumento de $316 \%$ de 2010 a 2019, com média de crescimento por ano de 17,1\% (Gráfico 1). 
Gráfico 1. Representação gráfica aproximada do quantitativo do número de gestantes diagnosticadas com sífilis (2010 a 2019). Sergipe, 2021.

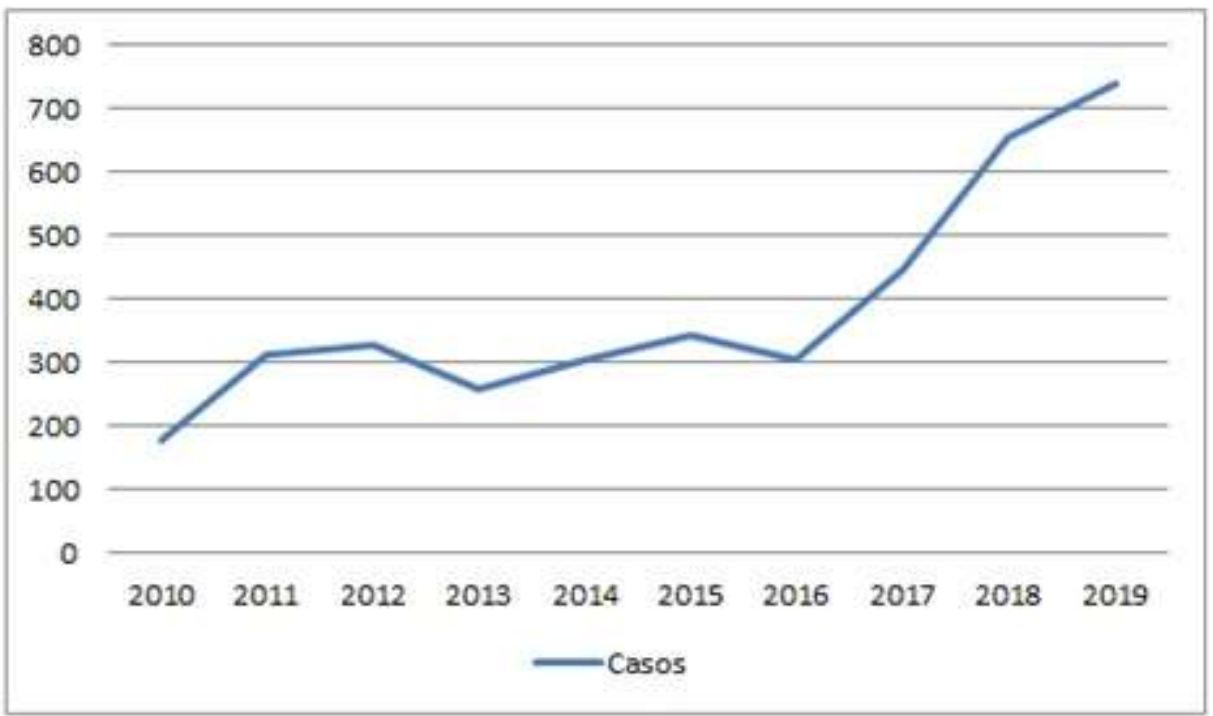

Fonte: Sistema Nacional de Agravos e Notificação. Secretaria de Vigilância em saúde, Ministério da Saúde, (2021).

Esse estudo identificou que, o maior número de casos ocorre entre gestantes de etnia autodeclarada como pardas. O total de mulheres pardas no período de 2010 a 2019 foi de 2.704 casos, com taxa de crescimento de $367 \%$ em dez anos, em comparação a 475 casos entre as mulheres brancas, 436 casos entre as mulheres pretas, 26 casos entre as mulheres amarelas, 13 casos em mulheres indígenas e 211 casos ignorados. As mulheres pardas representam cerca de 70,0\% do total de mulheres segundo a cor ou raça, além dos casos ignorados (Gráfico 2).

Gráfico 2. Representação gráfica aproximada da porcentagem do número de gestantes diagnosticadas com sífilis de acordo com cor ou raça (2010 a 2019).

Sergipe, 2021.

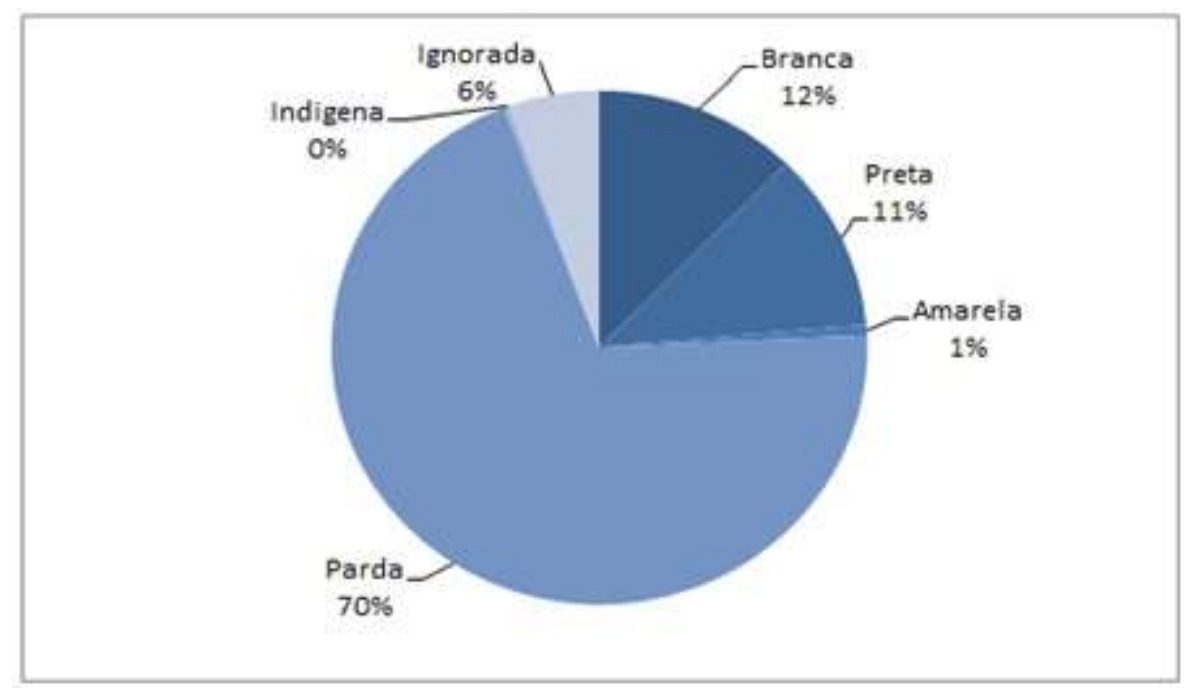

Fonte: Sistema Nacional de Agravos e Notificação. Secretaria de Vigilância em saúde, Ministério da Saúde, (2021). 
Em relação à faixa etária, notou-se um quantitativo expressivo entre a idade adulta jovem (20 a 29 anos). Assim, essa faixa etária correspondeu a 1.944 casos do total. Em seguida, 939 casos de mulheres entre 30 a 39 anos; e 830 casos de mulheres entre 15 a 19 anos. As faixas etárias com as menores prevalências foram as de maiores de 40 anos com 107 casos e as de 10 a 14 anos com 43 casos., nenhuma idade foi ignorada. Em porcentagens, o quantitativo de mulheres de 20 a 29 anos representa cerca de 50,3\% das mulheres nas faixas etárias estudadas (Gráfico 3).

Gráfico 3. Representação gráfica aproximada da porcentagem do número de gestantes diagnosticadas com sífilis de acordo com a faixa etária (2010 a 2019).

Sergipe, 2021.

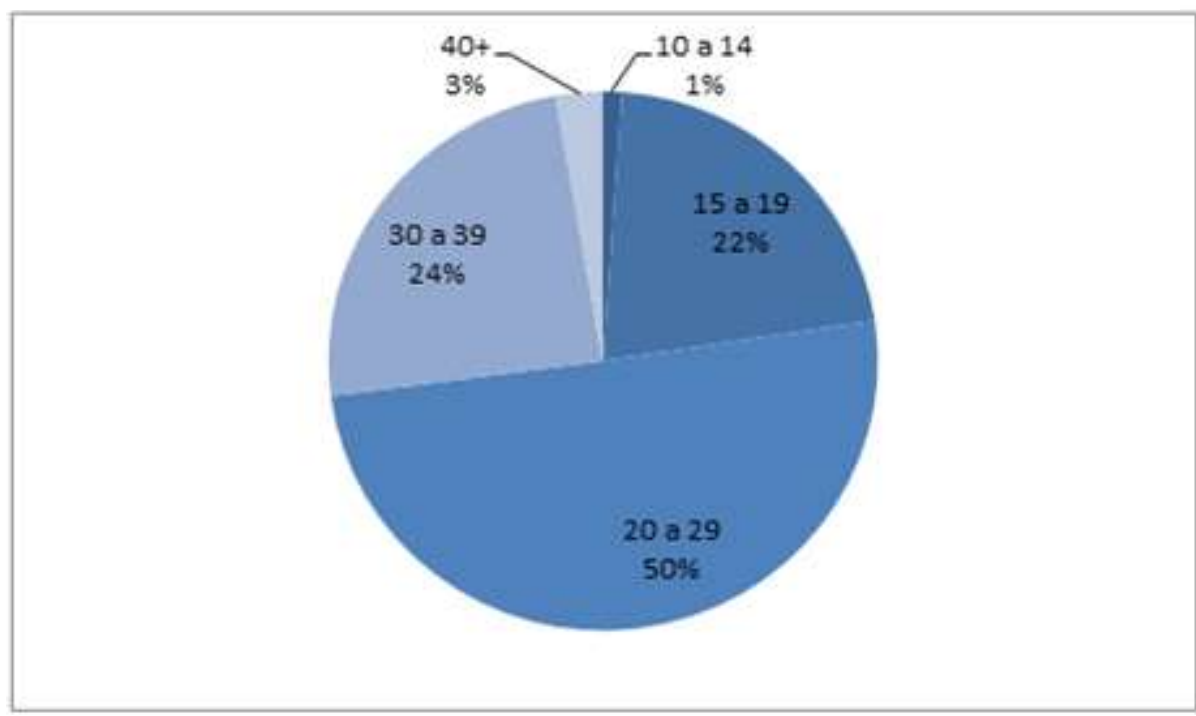

Fonte: Sistema Nacional de Agravos e Notificação. Secretaria de Vigilância em saúde, Ministério da Saúde, (2021).

Ademais, comparando a escolaridade entre as gestantes diagnosticadas, observou-se uma prevalência do quantitativo em mulheres que possuem da $5^{\mathrm{a}}$ a $8^{\mathrm{a}}$ série incompleta (Tabela 1). Assim sendo, dos 3.865 casos analisados, 1.045 correspondem a gestante como ensino fundamental incompleto (Tabela 2), o que equivale aproximadamente a $27 \%$ dos casos (Gráfico 4). 
Tabela 1. Casos de gestantes com sífilis segundo escolaridade por ano de diagnóstico. Sergipe, 20102019.

\begin{tabular}{|c|c|c|c|c|c|c|c|c|c|c|}
\hline Escolaridade & 2010 & 2011 & 2012 & 2013. & 2014 & 2015 & 2016 & 2017 & 2018 & 2019 \\
\hline Analfabeto & 7 & 11 & 5 & 6 & 10 & 5 & 6 & 8 & 6 & 3 \\
\hline 19 a 4 térie & & & & & & & & & & \\
\hline incompleta & 43 & 51 & 77 & 42 & 38 & 53 & 38 & 47 & 66 & 82 \\
\hline 41 sériecompleta & 11 & 37 & 29 & 22 & 22 & 28 & 21 & 27 & 48 & 31 \\
\hline 5îla 87 série & & & & & & & & & & \\
\hline incompleta & 38 & 50 & 86 & 64 & 74 & 75 & 64 & 124 & 210 & 260 \\
\hline $\begin{array}{l}\text { Fundamental } \\
\text { Completo }\end{array}$ & 8 & 17 & 13 & 15 & 22 & 32 & 29 & 46 & 66 & 63 \\
\hline Médio Incompleto & 6 & 19 & 21 & 25 & 30 & 36 & 30 & 46 & 82 & 102 \\
\hline Médio Completo & 7 & 31 & 20 & 29 & 26 & 37 & 42 & 67 & 92 & 76 \\
\hline Superior Incompleto & 4 & - & 3 & - & 5 & 3 & 1 & 8 & 3 & 8 \\
\hline Superior completo & 1 & 1 & 3 & 5 & 1 & 2 & 2 & 5 & 8 & 7 \\
\hline Năo se aplica & 1 & - & - & - & - & - & - & - & 1 & - \\
\hline Isnorado & 52 & 95 & 69 & 51 & 75 & 70 & 72 & 69 & 71 & 109 \\
\hline
\end{tabular}

Fonte: Sistema Nacional de Agravos e Notificação. Secretaria de Vigilância em saúde, Ministério da Saúde, (2021).

Tabela 2. Total de casos de gestantes com sífilis segundo escolaridade. Sergipe, 2021.

\begin{tabular}{lr}
\multicolumn{1}{c}{ Escolaridade } & Total \\
Analfabeto & 67 \\
$1 \geq$ a 4 a série incompleta & 537 \\
4 a série completa & 276 \\
5 a a 8 a série incompleta & 1045 \\
Fundamental Completo & 311 \\
Médio Incompleto & 397 \\
Médio Completo & 427 \\
Superior Incompleto & 35 \\
Superior Completo & 35 \\
Não se aplica & 2 \\
Ignorado & 733
\end{tabular}

Fonte: Sistema Nacional de Agravos e Notificação. Secretaria de Vigilância em saúde, Ministério da Saúde, (2021). 
Gráfico 4. Porcentagem do total de gestantes com sífilis segundo escolaridade Sergipe, 2021.

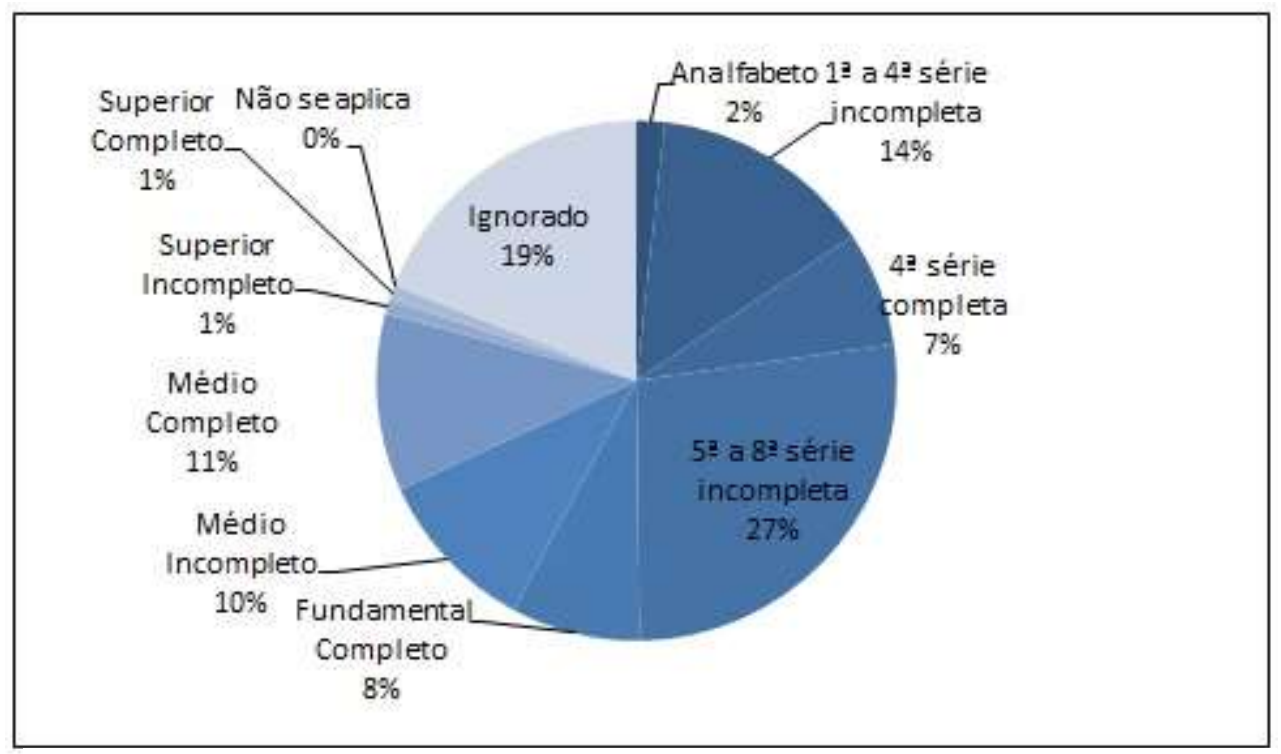

Fonte: Sistema Nacional de Agravos e Notificação. Secretaria de Vigilância em saúde, Ministério da Saúde, (2021).

No que se refere ao trimestre apresentado pelas gestantes no diagnóstico, das 3869 gestantes avaliadas, aproximadamente 38\% dos casos notificados (Gráfico 5) receberam o diagnóstico no terceiro trimestre gestacional. Ademais, 1371 mulheres receberam o diagnóstico no segundo trimestre e 927 no primeiro trimestre de gestação (Tabela 3).

Tabela 3. Casos de gestantes com sífilis segundo idade gestacional por ano de diagnóstico. Sergipe, 2021.

\begin{tabular}{|c|c|c|c|c|c|c|c|c|c|c|c|c|}
\hline & 2010 & & 2011 & 2012 & 2013 & 2014 & 2015 & 2016 & 2017 & 2018 & 2019 & Total \\
\hline $1^{*}$ Trimestre & & 22 & 35 & 44 & 46 & $\quad 71$ & 105 & $\quad 111$ & 155 & $5 \quad 174$ & 164 & $\quad 927$ \\
\hline $2^{\prime}$ Trimestre & & 53 & 132 & 127 & 133 & 156 & 152 & 131 & 149 & 165 & 173 & 1371 \\
\hline $3^{*}$ Trimestre & & 86 & 118 & 144 & 72 & 71 & 79 & 53 & 135 & 306 & 398 & 1462 \\
\hline $\begin{array}{l}\text { Idade } \\
\text { gestacional } \\
\text { ignorada }\end{array}$ & & 17 & 27 & 11 & 8 & 5 & 5 & 10 & 8 & 8 & 6 & 105 \\
\hline lgnorado & . & & - & - & . & - & . & - & . & . & . & 3869 \\
\hline
\end{tabular}

Fonte: Sistema Nacional de Agravos e Notificação. Secretaria de Vigilância em saúde, Ministério da Saúde, (2021). 
Gráfico 5. Representação gráfica aproximada da porcentagem do numero de gestantes diagnosticadas com sífilis segundo idade gestacional, 2021.

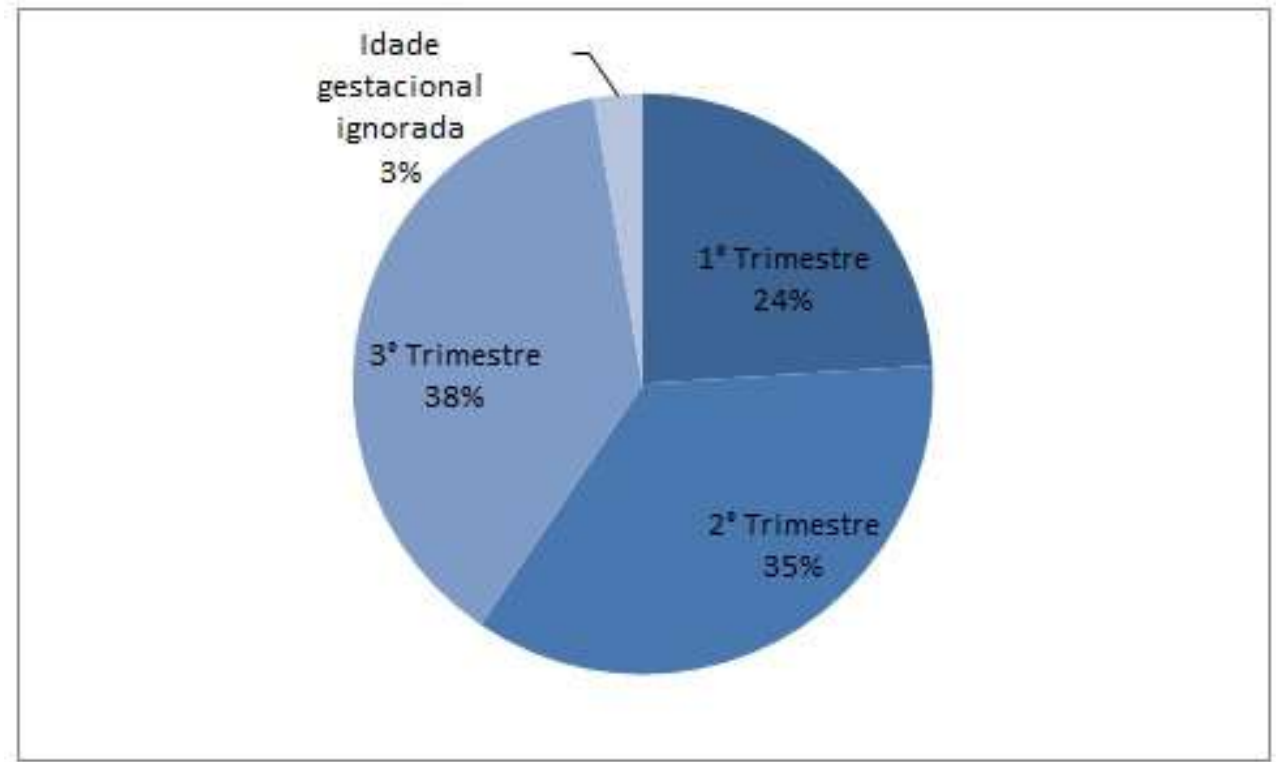

Fonte: Sistema Nacional de Agravos e Notificação Secretaria de Vigilância em saúde, Ministério da Saúde, (2021).

\section{Discussão}

Esta análise evidenciou significativo aumento no número de casos de sífilis em gestantes, no estado de Sergipe, no período de 2010 a 2019, que passou de 5,2 para 21,6/1.000 nascidos vivos. Além disso, mostrou maior prevalência dos casos em mulheres jovens de 20 a 29 anos, de etnia parda, com baixa escolaridade e com o maior número de diagnósticos no terceiro trimestre de gestação.

No Brasil, todas as mulheres grávidas têm o direito de realizar os exames de rastreio assegurados pela Portaria $\mathrm{N}^{\mathrm{o}}$ 2104, de 19 de novembro de 2002 do Ministério da Saúde, durante o pré-natal. Além disso, ocorreu em 2011 o início da implantação da Rede Cegonha em todos os estados brasileiros. Assim, é plausível supor que os serviços de saúde estão conseguindo maior captação e acompanhamento das gestantes e assim, tendo uma maior taxa de detecção da sífilis.

No país, como estratégias de diagnóstico de sífilis gestacional na atenção básica existem a triagem por meio do Venereal Disease Research Laboratory Test (VDRL) e o teste rápido (treponêmico) no primeiro e terceiro trimestres de gestação, com a consequente anotação clara e precisa dos resultados e do tratamento realizado. O acompanhamento preconizado requer que a gestante seja tratada, relatando esquema terapêutico e se esse tratamento foi adequado - esquema completo. Além disso, é de suma importância também a testagem do parceiro e o tratamento adequado.

Apesar da melhoria na cobertura pré-natal ao longo dos anos, apenas $20 \%$ das gestantes recebem cuidados adequados e, em muitos municípios, menos de $50 \%$ delas realizam duas sorologias para sífilis durante a gestação, conforme recomendação do Ministério da Saúde, o que gera atrasos no diagnóstico e inviabiliza o tratamento em tempo hábil (Domingues et al, 2015). O acesso, a utilização e a qualidade dos serviços de saúde para as mães são essenciais para garantir o bloqueio da transmissão vertical da sífilis (Pan American Health Organization, 2017), sendo fundamental para isso a captação precoce das gestantes e a realização do acompanhamento gestacional (Almeida, 2015). As mulheres com baixa frequência às 
consultas de pré-natal apresentam maior risco para a ocorrência da doença, efeito que diminui à medida que cresce o número de consultas (Macedo, et al., 2017).

Além da maior taxa de detecção por acompanhamento precoce, o aumento pode se dar por fatores sociodemográficos e de vulnerabilidade. Em um estudo de caso e controle feito em Recife (PE), foram identificados como fatores determinantes para a sífilis gestacional a ocorrência de apenas uma a três consultas no pré-natal e história anterior de infecção sexualmente transmissível, entre outros fatores que colocam a mulher em situação vulnerável (Macedo, et al., 2017).

Em uma pesquisa realizada com 2.422 gestantes em acompanhamento pré-natal em unidades de saúde da rede SUS do Rio de Janeiro nos anos 2007 e 2008, foi identificado 46 gestantes com sífilis e observou-se maior prevalência de sífilis na gestação em mulheres de cor preta, de menor classe econômica e escolaridade, que apresentavam antecedentes obstétricos de risco, assistência pré-natal realizada em UBS, início mais tardio do pré-natal e número inadequado de consultas (Domingues, et al., 2013). Em outro estudo multicêntrico nacional sobre soroprevalência da sífilis em puérperas realizado no período de 1999 a 2000, com uma amostra de 3.047 mulheres, mostrou que o maior risco para VDRL positivo estava associado, dentre outros fatores, à renda familiar, idade materna e baixa escolaridade (Rodrigues \& Guimarães, 2004).

Em estudo feito em Sergipe em 2009, foi detectada associação entre a idade e soroprevalência para testes de VDRL, observando-se maior positividade entre as gestantes com idade entre 20 a 34 anos (Inagaki, et al., 2009), estando ainda em conformidade com a faixa etária encontrada de 2010 a 2019. Essa mesma faixa etária foi encontrada pelo United States Centers for Disease Control and Prevention, em que nos Estados Unidos as taxas mais altas entre as mulheres estavam entre 20 a 24 anos de idade (11,6 casos por 100.000 mulheres).

Dados foram encontrados em países desenvolvidos como os Estados Unidos, em que a infecção é mais comum entre as mulheres grávidas pobres, jovens (idade $<29$ anos), negras e sem seguro de saúde e cuidados pré-natais. Outros fatores de risco incluem o uso de drogas ilícitas (por exemplo, metanfetamina, heroína), infecção com outras doenças sexualmente transmissíveis, residência em uma área de alta prevalência de sífilis, profissionais do sexo, ter mais de um parceiro sexual no ano passado, início do pré-natal no terceiro trimestre, encarceramento e moradia instável (CDC, 2021).

Dessa forma, a ocorrência de sífilis na gestação está associada à etnia parda, ao baixo nível de escolaridade, às condições socioeconômicas piores, aos antecedentes de risco obstétrico, ao início tardio do acompanhamento pré-natal, ao número insuficiente de consultas (Domingues, et al., 2013), às mulheres de idade jovem, além da baixa adesão ao pré-natal precoce e falha no tratamento.

Embora a sífilis seja uma doença para a qual existem recursos diagnósticos e terapêuticos simples e de baixo custo, seu controle na gestação mostra-se um desafio para profissionais de saúde e gestores. Isso em decorrência do curto intervalo da gestação para a realização do seu diagnóstico e tratamento; pela dificuldade de abordagem das doenças sexualmente transmissíveis, principalmente durante a gestação; e provavelmente pelo desconhecimento da magnitude desse agravo e dos danos que ele pode causar à saúde da mulher e do bebê pela população e pelos profissionais de saúde (Domingues, et al., 2013). Além disso, muitas mulheres ainda não têm acesso adequado aos serviços de saúde, assim acabam não realizando o teste de rastreio no pré-natal ou iniciando o acompanhamento tardiamente (Macedo, et al., 2017).

\section{Conclusão}

O resultado do presente estudo sugere que houve aumento considerável no número de casos notificados de sífilis na gestação, no estado de Sergipe e o perfil epidemiológico identificado é composto por mulheres jovens (20-34 anos), de etnia parda, com menos de 8 anos de escolaridade e com o diagnóstico da infecção no terceiro trimestre da gestação. Com isso, podemos inferir que a sífilis é uma importante doença prevalente na sociedade brasileira, em especial, no estado de Sergipe. 
Dessa maneira, a triagem pré-natal é fundamental para uma abordagem assertiva que previne agravos causados pela doença. Além disso, a ampliação da notificação dos casos de sífilis na gestação no SINAN, a busca sistemática de casos de sífilis congênita em todos os sistemas de informação e a melhoria do preenchimento das fichas de notificação e investigação são importantes para um melhor controle (Domingues et al, 2013). Assim sendo, a qualidade da assistência à gestação e parto é um importante determinante na redução das taxas de transmissão vertical da sífilis e o controle da doença tem como fundamento a triagem sorológica e o tratamento adequado de gestantes e parceiros (Campos, 2010) bem como o incentivo da educação sexual entre jovens e pessoas de baixa renda.

Espera-se que o estudo contribua para reformulação global das medidas no combate à Sífilis no estado de Sergipe, englobando desde o processo de triagem ao pré-natal até a finalização do tratamento com posterior controle dos títulos da sorologia do casal. Com base nos dados encontrados na pesquisa, sugere-se para trabalhos futuros, a verificação das medidas feitas para diminuir os casos de sífilis na gestação, bem como a verificação da prevalência das gestantes com diagnóstico de acordo com faixa etária, etnia, escolaridade e idade gestacional conforme o período escolhido pelo pesquisador, a fim de que, à partir dos resultados obtidos, seja possível planejar novas estratégias de combate a sífilis gestacional.

\section{Referências}

Almeida, P. D., Araújo Filho, A. C. A. Araújo, A . K. L., Carvalho, M. L., Silva, M. G. P., \& Araújo, T. M. E. (2015). Análise epidemiológica da sífilis congênita no Piauí. Revista Interdisciplinar; 8:62-70.

Arnesen, L., Martinez, G., Mainero, L., Serruya, S., \& Durán P. (2014). Gestational syphilis and stillbirth in Latin America and the Caribbean. Int J Gynaecol Obstet.;128 (3):241-5. doi:10.1016/j.ijgo.2014.09.017.

Cameron, C. E. \& Lukehart, S. A. (2014). Current status of syphilis vaccine development: need, challenges, prospects. Vaccine, 32(14):1602-9. doi:10.1016/j.vaccine.2013.09.053.

Campos, A. L. A., Araújo, M. A. L., Melo, S. P., \& Gonçalves, M. L. C. (2010). Epidemiologia da sífilis gestacional em Fortaleza, Brasil: um agravo sem controle. Cad Saúde Pública; 26:1747-56.

Casal, C. A. D., Silva, M. O., Costa, I. B., Araújo, E. C., \& Corvelo, T. C. O. (2011). Molecular detection of Treponema pallidum sp. Pallidum in blood samples of VDRL- seroreactive women with lethal pregnancy outcomes: a retrospective observational study in northern Brazil. Rev Soc Bras Med Trop.;44(4):451-6. doi:10.1590/S0037-86822011005000047.

Domingues, R. M. S. M. et al. (2013). Sífilis congênita: evento sentinela da qualidade da assistência pré-natal. Revista de Saúde Pública [online]. 47(1), 147157. doi:10.1590/S0034-89102013000100019

Domingues, R. M. S. M., Dias, M. A. B., Leal, M. C., Gama, S. G. N., Theme-Filha, M. M., \& Torres, J. A., et al. (2015). Adequacy of prenatal care according to maternal characteristics in Brazil. Rev Panam Salud Publica; 37(3):140-7. https://pubmed. ncbi.nlm.nih.gov/25988250/.

Estrela, C. (2018). Metodologia Científica: Ciência, Ensino, Pesquisa. Editora Artes Médicas.

Gomez, G. B., Kamb, M. L., Newman, L. M., Mark, J., Broutet, N., \& Hawkes, S. J. (2013). Untreated maternal syphilis and adverse outcomes of pregnancy: a systematic review and meta-analysis. Bull WHO. 91(3):217-26.:10.2471/BLT.12.107623.

Inagaki, A. D. M. et al. (2009). Soroprevalência de anticorpos para toxoplasmose, rubéola, citomegalovírus, sífilis e HIV em gestantes sergipanas. Revista da Sociedade Brasileira de Medicina Tropical. 42(5), 532-536. 10.1590/S0037-86822009000500010

Macedo, V. C. et al. (2017). Risk factors for syphilis in women: case-control study. Revista De Saúde Pública, 51, 78. 10.11606/s1518-8787.2017051007066

Musher, D. M., Holmes, K. K., Mardh, P. A., \& Sparling, P. F. et al. (1990). Biology of Treponema pallidum. In: Sexually Transmitted Diseases, McGrawHill,. p.205.

Pan American Health Organization. Elimination of mother-to-child transmission of HIV and syphilis in the Americas. (2017). Washington DC: Pan American Health Organization.

Rac, M. W., Revell, P. A., \& Eppes, C. S. (2017). Syphilis during pregnancy: a preventable threat to maternal-fetal health. Am J Obstet Gynecol, $216(4): 352$.

Rodrigues, C. S., \& Guimarães, M. D. C. (2004). Grupo Nacional de Estudo sobre Sífilis Congênita. Positividade para sífilis em puérperas: ainda um desafio para o Brasil. Rev Panam Salud Pública, 16:168-75.

Rowley, J., Hoorn, S. V., Korenromp, E., Low, N., Unemo, M., \& Abu-Raddad, L. et al. (2019). Chlamydia, gonorrhoea, trichomoniasis and syphilis: global prevalence and incidence estimates, 2016. Bull World Health Organ, 97:548-62.

Saraceni, V., \& Leal, M. C. (2003). Avaliação da efetividade das campanhas para eliminação da sífilis congênita na redução da morbi-mortalidade perinatal. Município do Rio de Janeiro, 1999-2000. Cadernos de Saúde Pública, 19(5):1341-1349. 
Research, Society and Development, v. 10, n. 13, e596101321617, 2021

(CC BY 4.0) | ISSN 2525-3409 | DOI: http://dx.doi.org/10.33448/rsd-v10i13.21617

Secretaria de Estado da Saúde. Plano Estadual de Saúde 2016-2019 (2016). https://www.conass.org.br/.

United States Centers for Disease Control and Prevention. Sexually Transmitted Disease Surveillance. (2019). https://www.cdc.gov/std/statistics/2019/default.htm.

United States Centers for Disease Control and Prevention. Sexually transmitted disease surveillance. (2015). http://www.cdc.gov/std/stats15/std-surveillance2015-print.pdf.

United States Centers for Disease Control and Prevention. Sexually transmitted disease surveillance. (2018). https://www.cdc.gov/std/stats18/Syphilis.htm. 